\title{
Concentration-Dependent Solvation Structure and
}

\author{
Dynamics of Aqueous Sulfuric Acid Using
}

Multinuclear NMR and DFT - Supporting

\section{Information}

J. David Bazak, Allison Wong, ${ }^{\dagger}$ Kaining Duanmu, Kee Sung Han, David Reed, Vijay

Murugesan*

Pacific Northwest National Laboratory, Richland, WA 99354, USA 


\section{Supporting Information}

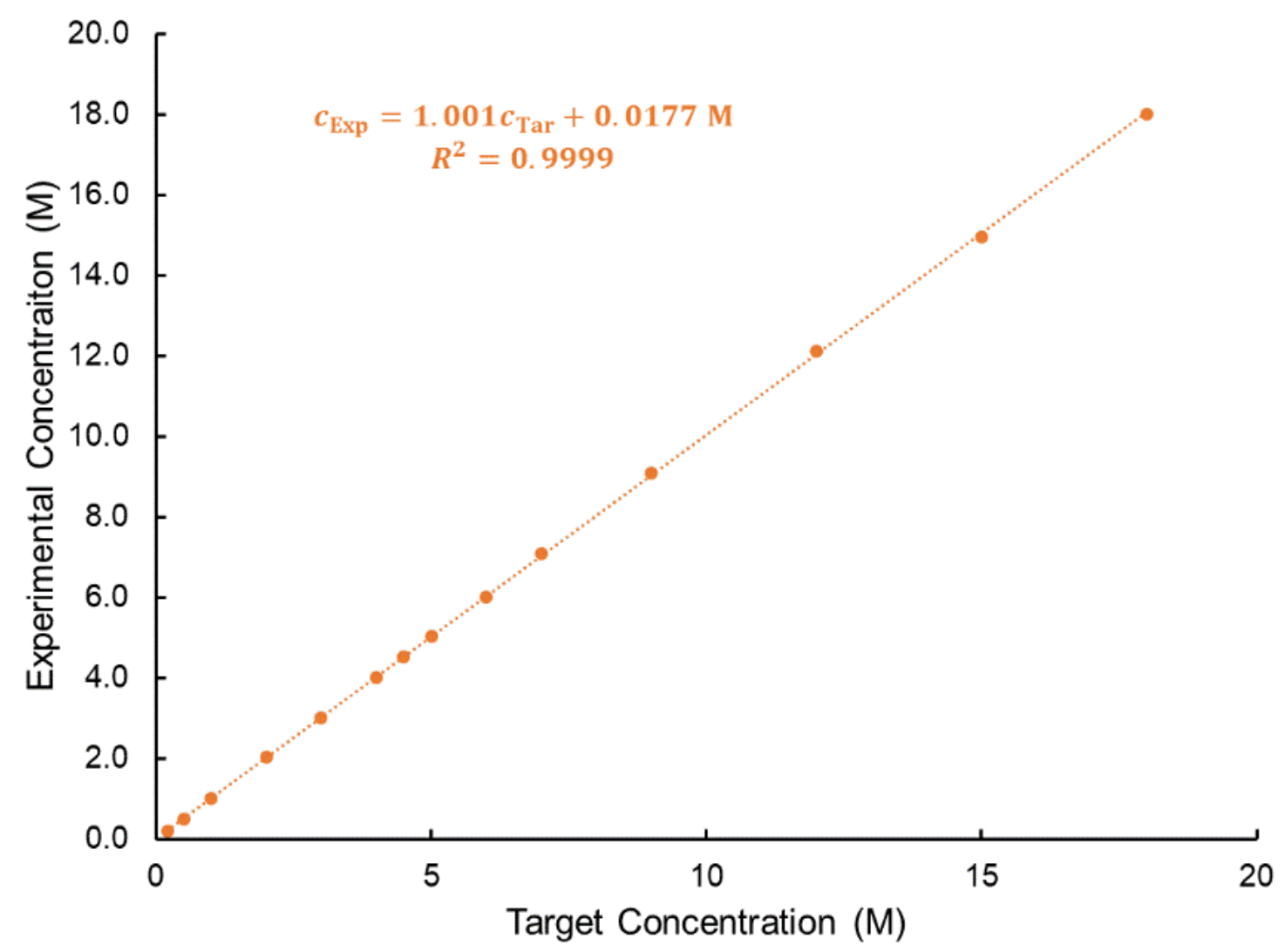

Figure S1. Correlation between the targeted $\mathrm{H}_{2} \mathrm{SO}_{4}$ (aq) concentrations and the experimentally determined concentrations from the added mass of $99.99 \% \mathrm{H}_{2} \mathrm{SO}_{4}$ and the volumetric flask volume, demonstrating the accuracy of the preparations. 

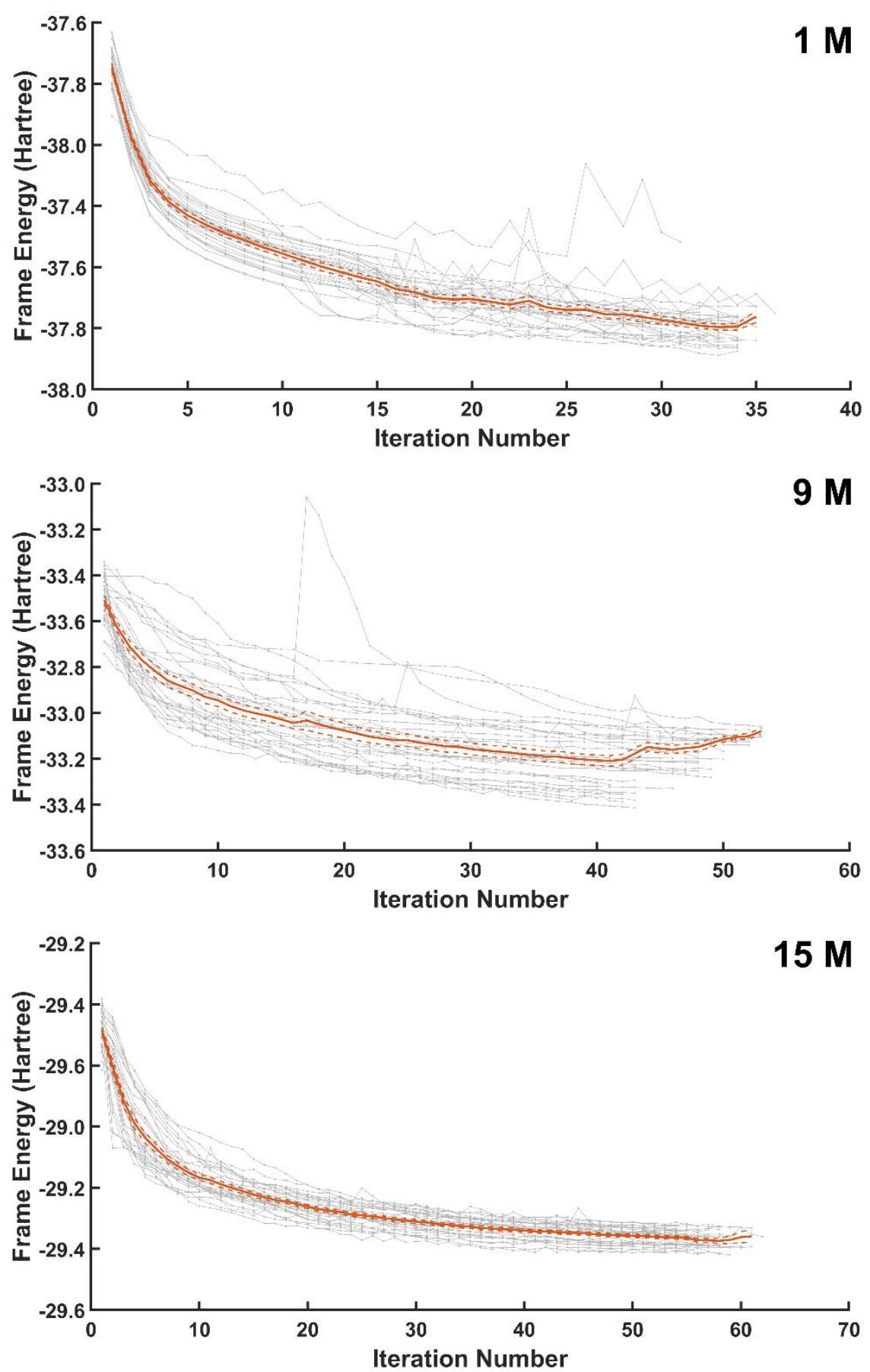

Figure S2. Frame energy convergence versus geometry optimization iteration number for all 30 frames in each of the target concentrations, demonstrating the stabilization of the total frame energy as the DFTB bonding is adjusted by the DFT geometry optimization. The orange line is the mean frame energy at each iteration and the accompanying dashed lines are the standard error, which merely serves as a guide to the eye. 


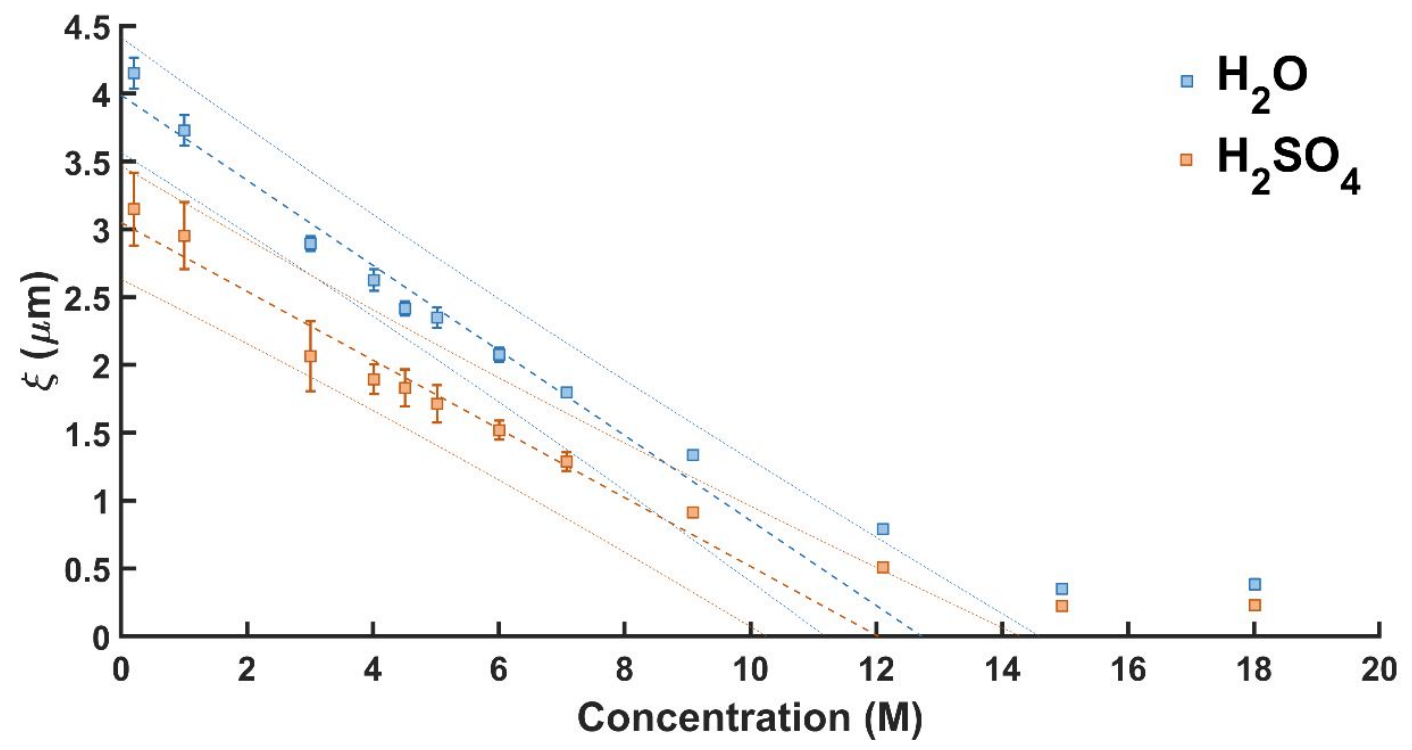

Figure S3. The characteristic proton diffusion length (root-mean-square displacement), $\xi=$ $\sqrt{D^{*}\left({ }^{1} \mathrm{H}\right) T_{1}\left({ }^{17} \mathrm{O}\right)}$ within the ${ }^{17} \mathrm{O}$ longitudinal relaxation time, for both sets of ${ }^{17} \mathrm{O} T_{1}$ values, at $298 \mathrm{~K}$. The linear extrapolations (dashed lines) are for all $\mathrm{H}_{2} \mathrm{SO}_{4}$ concentrations below $9 \mathrm{M}$ and fall to approximately zero in the concentration range where the secondary eutectic point lies in the $\mathrm{H}_{2} \mathrm{SO}_{4} / \mathrm{H}_{2} \mathrm{O}$ liquid-solid phase diagram. Dotted lines represent $95 \%$ prediction intervals. 

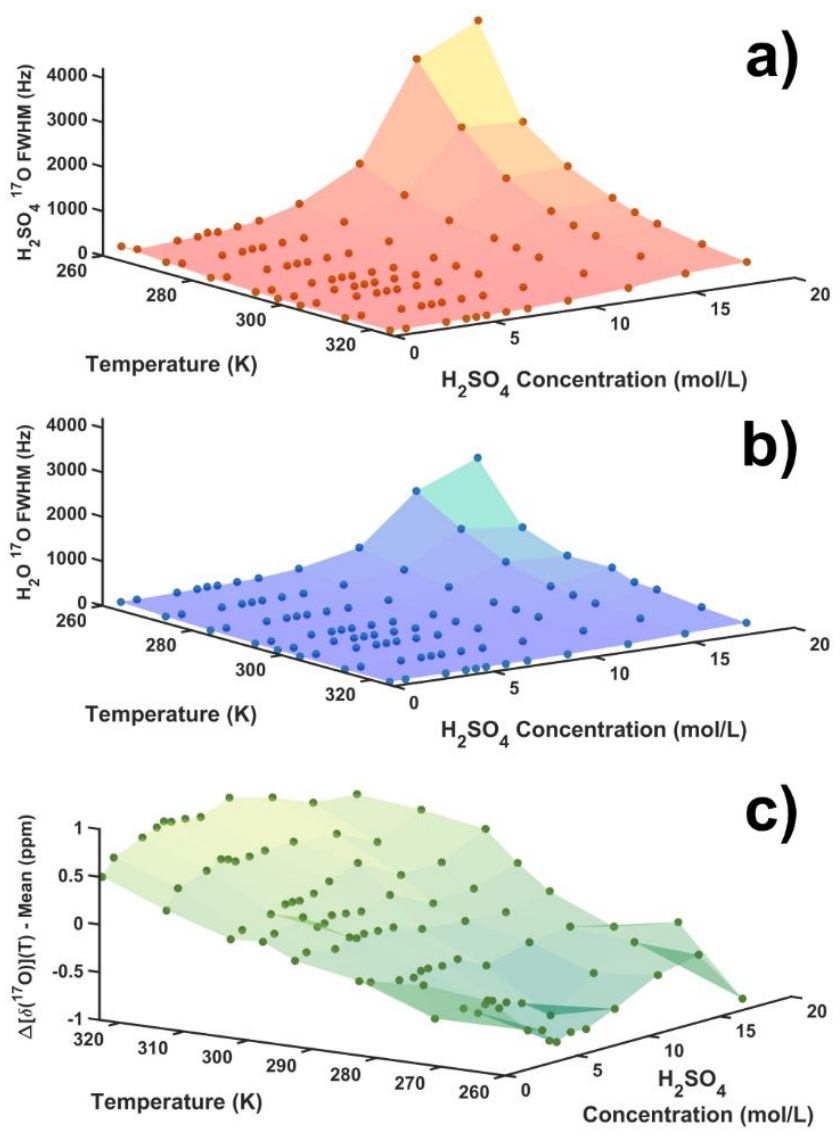

Figure S4. a) ${ }^{17} \mathrm{O} \mathrm{H}_{2} \mathrm{SO}_{4} / \mathrm{HSO}_{4}{ }^{-} / \mathrm{SO}_{4}{ }^{2-}$ linewidth, measured by the full-width-at-half-maximum (FWHM), as a function of both temperature and concentration, and b) similarly for $\mathrm{H}_{2} \mathrm{O} / \mathrm{H}_{3} \mathrm{O}^{+}$. c) The chemical shift difference at a given temperature, $\Delta\left[\delta\left({ }^{17} \mathrm{O}\right)\right](T)$, compared against the mean for all temperatures at a given concentration as a measure of deviation with temperature. The trend is small relative to the line shape fitting uncertainty (error bars would span most of the $\pm 1 \mathrm{ppm}$ vertical axis) but is nevertheless notable given the much larger chemical shift difference for low concentrations than high concentrations in absolute terms, which temperature evidently does not significantly affect. In all three cases, the interpolation surfaces function merely as a guide to the eye. 
Table S1. Complete data table for variable-temperature ${ }^{1} \mathrm{H}$ PFG-NMR self-diffusivity measurements across all studied concentrations. All values are in units of $10^{10} \mathrm{~m}^{2} / \mathrm{s}$.

\begin{tabular}{|l|c|c|c|c|c|c|c|c|}
\cline { 2 - 9 } \multicolumn{1}{c|}{} & $-10^{\circ} \mathrm{C}$ & $0^{\circ} \mathrm{C}$ & $10^{\circ} \mathrm{C}$ & $20^{\circ} \mathrm{C}$ & $25^{\circ} \mathrm{C}$ & $30^{\circ} \mathrm{C}$ & $40^{\circ} \mathrm{C}$ & $50^{\circ} \mathrm{C}$ \\
\hline $0.2 \mathrm{M}$ & & & $15.9 \pm 0.8$ & $21.6 \pm 0.9$ & $23.6 \pm 0.9$ & $28 \pm 1$ & $42 \pm 2$ & $84 \pm 2$ \\
\hline $1.0 \mathrm{M}$ & & $9.71 \pm 0.03$ & $13.6 \pm 0.2$ & $18.2 \pm 0.1$ & $20.7 \pm 0.2$ & $23.5 \pm 0.2$ & $32.4 \pm 0.3$ & $50.3 \pm 0.5$ \\
\hline $3.0 \mathrm{M}$ & $6.05 \pm 0.03$ & $7.35 \pm 0.04$ & $10.02 \pm 0.05$ & $13.5 \pm 0.1$ & $15.2 \pm 0.1$ & $17.1 \pm 0.1$ & $23.09 \pm 0.08$ & $33.9 \pm 0.2$ \\
\hline $4.0 \mathrm{M}$ & $4.98 \pm 0.03$ & $6.34 \pm 0.03$ & $8.97 \pm 0.07$ & $12.06 \pm 0.09$ & $13.8 \pm 0.1$ & $15.7 \pm 0.2$ & $21.6 \pm 0.2$ & $29.1 \pm 0.4$ \\
\hline $4.5 \mathrm{M}$ & $4.66 \pm 0.07$ & $5.98 \pm 0.02$ & $8.1 \pm 0.2$ & $10.9 \pm 0.1$ & $12.4 \pm 0.2$ & $13.9 \pm 0.1$ & $18.23 \pm 0.09$ & $24.5 \pm 0.2$ \\
\hline $5.0 \mathrm{M}$ & & $5.39 \pm 0.03$ & $7.58 \pm 0.03$ & $10.13 \pm 0.06$ & $11.7 \pm 0.1$ & $13.2 \pm 0.1$ & $17.7 \pm 0.1$ & $24.86 \pm 0.03$ \\
\hline $6.0 \mathrm{M}$ & $3.41 \pm 0.04$ & $4.98 \pm 0.09$ & $6.96 \pm 0.09$ & $9.2 \pm 0.1$ & $10.5 \pm 0.1$ & $11.9 \pm 0.2$ & $15.1 \pm 0.2$ & $21.2 \pm 0.3$ \\
\hline $7.0 \mathrm{M}$ & $2.81 \pm 0.04$ & $4.13 \pm 0.02$ & $5.63 \pm 0.08$ & $7.7 \pm 0.1$ & $8.7 \pm 0.1$ & $9.9 \pm 0.1$ & $13.1 \pm 0.1$ & $17.8 \pm 0.1$ \\
\hline $9.0 \mathrm{M}$ & & $1.86 \pm 0.03$ & $3.75 \pm 0.07$ & $5.23 \pm 0.05$ & $5.9 \pm 0.2$ & $6.9 \pm 0.1$ & $8.89 \pm 0.08$ & $11.4 \pm 0.1$ \\
\hline $12.0 \mathrm{M}$ & & $1.41 \pm 0.01$ & $1.94 \pm 0.02$ & $2.72 \pm 0.01$ & $3.21 \pm 0.03$ & $3.71 \pm 0.04$ & $5.15 \pm 0.07$ & $7.06 \pm 0.06$ \\
\hline $15.0 \mathrm{M}$ & & & $0.79 \pm 0.04$ & $1.1 \pm 0.3$ & $1.23 \pm 0.06$ & $1.5 \pm 0.2$ & $2.2 \pm 0.2$ & $3.1 \pm 0.3$ \\
\hline $18.0 \mathrm{M}$ & & & $0.82 \pm 0.01$ & $1.20 \pm 0.01$ & $1.46 \pm 0.01$ & $1.72 \pm 0.01$ & $2.38 \pm 0.03$ & $3.08 \pm 0.02$ \\
\hline
\end{tabular}

Table S2. Complete data table for variable-temperature ${ }^{17} \mathrm{O} \mathrm{T}_{1}$ measurements for the $\mathrm{H}_{2} \mathrm{O} / \mathrm{H}_{3} \mathrm{O}^{+}$signal across all studied concentrations. All values are in units of $\mathrm{ms}$.

\begin{tabular}{|l|c|c|c|c|c|c|c|c|}
\cline { 2 - 9 } \multicolumn{1}{c|}{} & $-10^{\circ} \mathrm{C}$ & $0^{\circ} \mathrm{C}$ & $10^{\circ} \mathrm{C}$ & $20^{\circ} \mathrm{C}$ & $25^{\circ} \mathrm{C}$ & $30^{\circ} \mathrm{C}$ & $40^{\circ} \mathrm{C}$ & $50^{\circ} \mathrm{C}$ \\
\hline $0.2 \mathrm{M}$ & $2.19 \pm 0.05$ & $3.12 \pm 0.06$ & $4.6 \pm 0.1$ & $6.16 \pm 0.04$ & $7.3 \pm 0.3$ & $8.5 \pm 0.3$ & $10.2 \pm 0.4$ & $12.4 \pm 0.3$ \\
\hline $1.0 \mathrm{M}$ & $2.12 \pm 0.05$ & $3.21 \pm 0.06$ & $4.5 \pm 0.1$ & $5.7 \pm 0.3$ & $6.7 \pm 0.4$ & $7.7 \pm 0.3$ & $9.6 \pm 0.4$ & $12 \pm 1$ \\
\hline $3.0 \mathrm{M}$ & $1.80 \pm 0.09$ & $2.57 \pm 0.09$ & $3.59 \pm 0.07$ & $4.9 \pm 0.2$ & $5.5 \pm 0.2$ & $6.1 \pm 0.2$ & $7.9 \pm 0.2$ & $9.9 \pm 0.5$ \\
\hline $4.0 \mathrm{M}$ & $1.65 \pm 0.03$ & $2.37 \pm 0.09$ & $3.4 \pm 0.1$ & $4.4 \pm 0.1$ & $5.0 \pm 0.3$ & $5.6 \pm 0.3$ & $7.0 \pm 0.3$ & $8.4 \pm 0.4$ \\
\hline $4.5 \mathrm{M}$ & $1.52 \pm 0.06$ & $2.3 \pm 0.1$ & $3.2 \pm 0.1$ & $4.3 \pm 0.2$ & $4.7 \pm 0.2$ & $5.4 \pm 0.2$ & $6.9 \pm 0.6$ & $8.1 \pm 0.6$ \\
\hline $5.0 \mathrm{M}$ & $1.54 \pm 0.06$ & $2.25 \pm 0.08$ & $3.07 \pm 0.07$ & $4.1 \pm 0.1$ & $4.7 \pm 0.3$ & $5.1 \pm 0.2$ & $6.5 \pm 0.4$ & $7.8 \pm 0.3$ \\
\hline $6.0 \mathrm{M}$ & $1.38 \pm 0.05$ & $2.0 \pm 0.1$ & $2.8 \pm 0.1$ & $3.6 \pm 0.2$ & $4.1 \pm 0.2$ & $4.8 \pm 0.2$ & $5.7 \pm 0.3$ & $7.1 \pm 0.5$ \\
\hline $7.0 \mathrm{M}$ & $1.33 \pm 0.06$ & $1.83 \pm 0.06$ & $2.60 \pm 0.06$ & $3.4 \pm 0.1$ & $3.7 \pm 0.1$ & $4.2 \pm 0.2$ & $5.4 \pm 0.3$ & $6.4 \pm 0.4$ \\
\hline $9.0 \mathrm{M}$ & $1.00 \pm 0.03$ & $1.44 \pm 0.04$ & $2.1 \pm 0.1$ & $2.56 \pm 0.08$ & $3.0 \pm 0.1$ & $3.4 \pm 0.2$ & $4.4 \pm 0.3$ & $5.3 \pm 0.3$ \\
\hline $12.0 \mathrm{M}$ & $0.56 \pm 0.02$ & $0.82 \pm 0.04$ & $1.20 \pm 0.08$ & $1.7 \pm 0.1$ & $1.9 \pm 0.1$ & $2.19 \pm 0.09$ & $3.1 \pm 0.2$ & $3.7 \pm 0.3$ \\
\hline $15.0 \mathrm{M}$ & $0.23 \pm 0.05$ & $0.31 \pm 0.02$ & $0.52 \pm 0.07$ & $0.9 \pm 0.1$ & $1.0 \pm 0.1$ & $1.3 \pm 0.1$ & $1.7 \pm 0.2$ & $1.7 \pm 0.2$ \\
\hline $18.0 \mathrm{M}$ & $0.4 \pm 0.2$ & $0.3 \pm 0.1$ & $0.6 \pm 0.3$ & $0.8 \pm 0.2$ & $1.0 \pm 0.2$ & $1.1 \pm 0.3$ & $1.5 \pm 0.2$ & $1.9 \pm 0.4$ \\
\hline
\end{tabular}


Table S3. Complete data table for variable-temperature ${ }^{17} \mathrm{O} \mathrm{T}_{1}$ measurements for the $\mathrm{H}_{2} \mathrm{SO}_{4} / \mathrm{HSO}_{4}{ }^{-} / \mathrm{SO}_{4}{ }^{2-}$ signal across all studied concentrations. All values are in units of $\mathrm{ms}$.

\begin{tabular}{|l|c|c|c|c|c|c|c|c|}
\cline { 2 - 9 } \multicolumn{1}{c|}{} & $-10^{\circ} \mathrm{C}$ & $0^{\circ} \mathrm{C}$ & $10^{\circ} \mathrm{C}$ & $20^{\circ} \mathrm{C}$ & $25^{\circ} \mathrm{C}$ & $30^{\circ} \mathrm{C}$ & $40^{\circ} \mathrm{C}$ & $50^{\circ} \mathrm{C}$ \\
\hline $0.2 \mathrm{M}$ & $1.7 \pm 0.7$ & $1.4 \pm 0.7$ & $2.3 \pm 0.7$ & $3.6 \pm 0.7$ & $4.2 \pm 0.7$ & $4.1 \pm 0.4$ & $4.7 \pm 0.5$ & $6.3 \pm 0.9$ \\
\hline $1.0 \mathrm{M}$ & $1.4 \pm 0.3$ & $2.1 \pm 0.4$ & $2.8 \pm 0.3$ & $3.5 \pm 0.6$ & $4.2 \pm 0.7$ & $4.4 \pm 0.4$ & $5.2 \pm 0.5$ & $6.3 \pm 0.9$ \\
\hline $3.0 \mathrm{M}$ & $1.3 \pm 0.2$ & $1.7 \pm 0.2$ & $2.2 \pm 0.2$ & $3.0 \pm 0.5$ & $2.8 \pm 0.7$ & $3.4 \pm 0.5$ & $4.0 \pm 0.4$ & $5.2 \pm 0.6$ \\
\hline $4.0 \mathrm{M}$ & $1.2 \pm 0.1$ & $1.6 \pm 0.2$ & $2.2 \pm 0.2$ & $2.6 \pm 0.4$ & $2.6 \pm 0.3$ & $3.5 \pm 0.4$ & $3.8 \pm 0.5$ & $5.3 \pm 0.6$ \\
\hline $4.5 \mathrm{M}$ & $1.1 \pm 0.1$ & $1.7 \pm 0.2$ & $2.1 \pm 0.2$ & $2.8 \pm 0.4$ & $2.7 \pm 0.4$ & $3.2 \pm 0.4$ & $3.4 \pm 0.7$ & $4 \pm 1$ \\
\hline $5.0 \mathrm{M}$ & $1.1 \pm 0.1$ & $1.5 \pm 0.2$ & $1.8 \pm 0.3$ & $2.5 \pm 0.4$ & $2.5 \pm 0.4$ & $3.2 \pm 0.3$ & $3.3 \pm 0.5$ & $4.0 \pm 0.6$ \\
\hline $6.0 \mathrm{M}$ & $0.95 \pm 0.06$ & $1.1 \pm 0.1$ & $1.5 \pm 0.2$ & $1.9 \pm 0.2$ & $2.2 \pm 0.2$ & $2.5 \pm 0.2$ & $3.0 \pm 0.3$ & $3.7 \pm 0.3$ \\
\hline $7.0 \mathrm{M}$ & $0.79 \pm 0.04$ & $1.07 \pm 0.07$ & $1.37 \pm 0.09$ & $1.6 \pm 0.2$ & $1.9 \pm 0.2$ & $2.1 \pm 0.2$ & $2.7 \pm 0.3$ & $3.2 \pm 0.3$ \\
\hline $9.0 \mathrm{M}$ & $0.50 \pm 0.02$ & $0.71 \pm 0.06$ & $0.93 \pm 0.06$ & $1.29 \pm 0.08$ & $1.4 \pm 0.1$ & $1.5 \pm 0.1$ & $2.0 \pm 0.1$ & $2.4 \pm 0.2$ \\
\hline $12.0 \mathrm{M}$ & $0.22 \pm 0.02$ & $0.38 \pm 0.05$ & $0.51 \pm 0.04$ & $0.71 \pm 0.03$ & $0.80 \pm 0.05$ & $0.89 \pm 0.04$ & $1.16 \pm 0.07$ & $1.43 \pm 0.04$ \\
\hline $15.0 \mathrm{M}$ & $0.12 \pm 0.04$ & $0.15 \pm 0.02$ & $0.26 \pm 0.04$ & $0.34 \pm 0.03$ & $0.41 \pm 0.03$ & $0.47 \pm 0.03$ & $0.68 \pm 0.08$ & $0.89 \pm 0.06$ \\
\hline $18.0 \mathrm{M}$ & $0.07 \pm 0.02$ & $0.16 \pm 0.03$ & $0.23 \pm 0.02$ & $0.31 \pm 0.03$ & $0.36 \pm 0.02$ & $0.44 \pm 0.03$ & $0.56 \pm 0.03$ & $0.80 \pm 0.05$ \\
\hline
\end{tabular}

